\title{
PIK3CA oncogenic mutations represent a major mechanism of resistance to trastuzumab in HER2/neu overexpressing uterine serous carcinomas
}

Jonathan D Black, Salvatore Lopez, Emiliano Cocco, Stefania Bellone, Gary Altwerger, Carlton L Schwab, Diana P English, Elena Bonazzoli, Federica Predolini, Francesca Ferrari, Elena Ratner, Dan-Arin Silasi, Masoud Azodi, Peter E Schwartz and Alessandro D Santin

Correction to: British Journal of Cancer (2015) 113, 1020-1026; doi:10.1038/ bjc. 2015.306

label above the figure reads as 'ARK-2 E93Q'. It should be corrected to 'ARK-2 R93Q'.

It has been brought to our attention that there is an error in Figure 3 of the above paper published in advance online on 1 September 2015. The original

\section{Benzene exposure and risk of lymphohaematopoietic cancers in $\mathbf{2 5 0 0 0}$ offshore oil industry workers}

J S Stenehjem, K Kjærheim, M Bråtveit, S O Samuelsen, F Barone-Adesi, N Rothman, Q Lan and T K Grimsrud

Correction to: British Journal of Cancer (2015) 112, 1603-1612; doi:10.1038/ bjc. 2015.108

After the publication of 'Benzene exposure and risk of lymphohaematopoietic cancers in 25000 offshore oil industry workers', the author would like to make the following corrections:

When referring to work by other researchers we used the symbol ' $<$ ' incorrectly. The expression '<0.2 p.p.m.' (reading: 'less than 0.2 p.p.m.') should have been expressed as 'down to 0.2 p.p.m.
This error occurs at two places in the article:

(1) Introduction, p. 1604, 1st column, 4th paragraph: 'Some reports have suggested that benzene may be leukaemogenic and haematotoxic at low concentrations ( $<10$ p.p.m. and $<0.2$ p.p.m., respectively).'

(2) Discussion, p. 1610, 2nd column, 3rd paragraph: 'Interestingly, the observed risk patterns for MM and CLL correspond to the finding of benzene toxicity to B-cells in the bone marrow at occupational benzene exposure $<0.2$ p.p.m.

Tumour expression of leptin is associated with chemotherapy resistance and therapy-independent prognosis in gastro-oesophageal adenocarcinomas

G H Bain, E Collie-Duguid, G I Murray, F J Gilbert, A Denison, F Mckiddie, T Ahearn, I Fleming, J Leeds, P Phull, K Park,

S Nanthakumaran, H I Grabsch, P Tan, A Welch, L Schweiger, A Dahle-Smith, G Urquhart, M Finegan, K M Matula and R D Petty

Correction to: British Journal of Cancer (2014) 110, 1525-1534; doi:10.1038/

bjc. 2014.45

Upon publication of the above paper in British Journal of Cancer, the authors noted that they had omitted KM Matula from the author list. The full and correct author listing is reproduced above.

(c) 2015 Cancer Research UK. All rights reserved 0007-0920/15

Comment on 'Are the studies on cancer risk from CT scans biased by indication? Elements of answer from a large-scale cohort study in France' - Evidence of confounding by predisposing factors unclear

E Cardis and M Bosch de Basea

Correction to: British Journal of Cancer (2015) 112, 1842-1843; doi:10.1038/ bjc. 2015.155

It has come to our attention that an author on Comment on: 'Are the studies factors unclear' does not have their name displayed on the paper correctly. The author's name is shown as MB de Basea when in fact it should be M Bosch de Basea. on cancer risk from CT scans biased by indication? Elements of answer from a 